\title{
LOS PAÍSES DEL TERCER MUNDO FRENTE A LA CRISIS EUROPEA*
}

\author{
PEDRO NEL PÁEZ $Z^{* *}$, JORGE ISAZA*** \& LUZ AMANDA ZAMORA**** \\ UNIVERSIDAD MILITAR NUEVA GRANADA \\ Recibido/ Received/ Recebido: 06/11/2013 - Aceptado/ Accepted/ Aprovado: 13/05/2014
}

\begin{abstract}
Resumen
El objetivo de este documento es determinar los canales de transmisión de la crisis europea hacia los países del Tercer Mundo. El trabajo examina la forma en que la crisis de la deuda soberana se propagó por el sector financiero, a través de la mayor volatilidad de la rentabilidad de los activos. Lo cual se debió a una mayor variación de sus precios, lo que tuvo efectos sobre el patrimonio neto de los agentes económicos, los ingresos, la demanda agregada y la asignación del crédito, así como también sobre la producción y el empleo. Para este documento se hizo búsqueda y selección de literatura especializada y se extrajeron estadísticas especializadas de la UNCTAD, CEPAL y OECD. Se concluye que la transmisión de la crisis europea al Tercer Mundo se dio por canales específicos conocidos, como el comercio internacional, la inversión extranjera directa, las remesas y la ayuda oficial para el desarrollo.
\end{abstract}

Palabras clave: Crisis soberana, Canales de transmisión, Patrimonio neto, Canal comercial y financiero, Sector real.

\section{THIRD WORLD COUNTRIES FACING EUROPEAN CRISIS}

\begin{abstract}
This paper identifies transmission pathways of European crisis to the Third World countries. The paper examines how sovereign debt crisis spread across financial sector, through the major volatility asset return. This was a result of higher prices change, which had an impact on net heritage of economic agents, income, aggregate demand, credit allocation, as well as on production and employment. This document includes specialized literature and statistics of UNCTAD, CEPAL and OECD. It is concluded that European crisis transmission to Third World countries was given by specific pathways known as international trade, direct foreign investment, remittances and official assistance for development.
\end{abstract}

Keywords: Sovereign crisis, Transmission channels, Net heritage, Commercial and financial channel, Real sector.

Informe final de la investigación "La Crisis de la Deuda en la Zona Euro: los Mecanismos de Transmisión y Efectos en el Tercer Mundo" VICEIN-R-014, Centro de Investigaciones FARIES-UMNG.

** PhD, Economía. Profesor Asociado, Escuela Superior de Administración Pública, ESAP. Correo electrónico: pedronelpaez@gmail. com

*** Profesor Asociado de la Facultad de Relaciones Internacionales, Estrategia y Seguridad de la Universidad Militar Nueva Granada. Correo electrónico: jorge.isaza@unimilitar.edu.co

***** PhD. Mg. Relaciones y Negocios Internacionales, UMNG. Co-investigadora Universidad Militar Nueva Granada. Correo electrónico: luzamanda511@hotmail.com 


\title{
OS PAÍSES DO TERCEIRO MUNDO FRENTE À CRISE EUROPEIA
}

\begin{abstract}
Resumo
O objetivo deste documento é determinar os canais de transmissão da crise europeia para os países do terceiro mundo. $\mathrm{O}$ trabalho examina a forma em que a crise da dívida soberana se propagou pelo setor financeiro, através da maior volatilidade da rentabilidade dos ativos. Isto se deveu por uma maior variação de seus preços, o que teve efeitos sobre o patrimônio líquido dos agentes econômicos, sobre os rendimentos, sobre a demanda agregada e sobre a atribuição do crédito, como também sobre a produção e o emprego. Para este documento se fez busca e seleção de literatura especializada e se extraíram estatísticas especializadas da UNCTAD, CEPAL e OECD. Conclui-se que a transmissão da crise europeia ao terceiro mundo se deu por canais específicos conhecidos como o comércio internacional, o investimento estrangeiro direto, as remessas e a ajuda oficial para o desenvolvimento.
\end{abstract}

Palavras chave: Crise soberana, Canais de transmissão, Patrimônio líquido, Canal comercial e financeiro, Setor real.

Páez, P., Isaza, J. \& Zamora, L. (2014) Los Países del Tercer Mundo Frente a la Crisis Europea. En: Revista de la Facultad de Ciencias Económicas de la Universidad Militar Nueva Granada. rev.fac. cienc.econ, XXII (1).

JEL: F19, F49.

\section{Introducción}

El propósito de esta investigación es determinar los canales por medio de los cuales la crisis de la deuda europea se propaga por el Tercer Mundo. El tema es relevante porque es un acontecimiento económico lamentable pero poco frecuente por la magnitud que ha alcanzado y porque se da en un entorno de países donde la renta per cápita es elevada con respecto al promedio mundial. Derivado de la crisis se ha llegado a situaciones sociales igualmente lastimosas, en las que una considerable cantidad de personas ha perdido sus empleos y las fuentes de ingresos, de manera que los niveles de bienestar se han afectado dramáticamente. Simultáneamente los gobiernos de la Unión, sin excepciones, han tenido que adelantar programas de austeridad severos, lo que en lenguaje llano significa que tienen que restringir el gasto público. En estas circunstancias equivale a disminuir los recursos destinados a programas de bienestar social. Estos elementos junto con la disminución o congelamiento de los salarios, implica profundos costos sociales. De manera la crisis europea, al igual que ha sucedido con otras crisis, tiene efectos múltiples pero devastadores sobre la producción, el empleo, el comercio internacional, las remesas y la ayuda internacional para el desarrollo de los países más pobres. Ante esa circunstancia, es lógico preguntarse si existen algunas formas específicas que afecten a los países del Tercer Mundo por la supuesta vulnerabilidad de estas sociedades a los vaivenes de las economías desarrolladas, a través de algunos canales de transmisión específicos que es necesario entender. La propagación y amplificación de las perturbaciones del sector financiero hacia la inversión productiva tiene consecuencias macroeconómicas que provienen precisamente de las alteraciones del sistema financiero. Cualquier efecto marginal de la crisis puede tener manifestaciones amplificadas y funestas para el Tercer Mundo.

Por tanto, el documento se ha estructurado de la siguiente manera. La sección 2 explora la relación entre la crisis de la deuda que vivió América Latina durante los años 80 y la crisis soberana de Europa. En ese sentido, los efectos sobre la producción y el desempleo de ésta última. La sección 3 analiza la forma concreta en que la crisis se transmite al mismo 
sector financiero y al sector real a través de la mayor volatilidad de la rentabilidad de los activos, producida esta en un aumento de la volatilidad de los precios. Explora también la transmisión de la crisis financiera a través del patrimonio neto de los agentes económicos, efectos negativos sobre los ingresos y de allí a la demanda agregada cuando se contraen los ingresos de los hogares y los beneficios de las empresas. La cuarta sección aborda el problema de transmisión de la crisis europea al Tercer Mundo a través de algunos canales específicos como el comercio internacional, la inversión extranjera directa, las remesas y la ayuda oficial que la Unión Europea destina a apoyar algunas actividades en los países no desarrollados. Metodológicamente la investigación se apoyó en fuentes secundarias y primarias. Las primeras están representadas por la búsqueda y selección de literatura especializada. Las últimas están representadas por las estadísticas especializadas, publicadas por organizaciones como la UNCTAD, CEPAL y OECD. Esta información permite seleccionar las variables relevantes para la investigación, como el comercio exterior, las remesas y la ayuda internacional. La última sección recoge las principales conclusiones del trabajo. Este es el segundo informe de la investigación "La Crisis de la Deuda en la Zona Euro: los Mecanismos de Transmisión y Efectos en el Tercer Mundo", auspiciado por el Centro de Investigaciones de la Facultad de Relaciones Internacionales, Estrategia y Seguridad de la Universidad Militar Nueva Granada.

\section{La Crisis Europea y el sector real: antecedentes}

La Unión Europea atraviesa por una crisis económica y social severa y persistente, amplificada por las duras medidas de austeridad impuestas a su población. Con la crisis financiera global de 20072009 , originada ella misma en la creciente liquidez en los mercados internacionales y el posterior estallido de la burbuja inmobiliaria en Estados Unidos, los países de la periferia europea (Grecia, Portugal, Italia, España e Irlanda) experimentaron un auge del consumo interno con un sesgo anti-exportador, que se tradujo en una pérdida de competitividad. Estos eventos originaron el aumento de la deuda pública y privada. Cuando no pudo pagarse, sobrevino no sólo la crisis de deuda, igualmente una crisis bancaria, el desplome de la producción y el constante aumento del desempleo.

El proceso implicó rescatar a Grecia y Portugal por el Banco Central Europeo (BCE) y el Fondo Monetario Internacional (FMI). Pero aún con la implementación de las medidas de austeridad y los recursos provenientes de las instituciones crediticias supranacionales, el Viejo Continente no logra superar esta condición crítica.

El escenario europeo genera interrogantes sobre la forma en que estas perturbaciones económicas y financieras se difunden a otras regiones, particularmente del Tercer Mundo, por el supuesto de una mayor vulnerabilidad a las inestabilidades observadas en el centro del mundo desarrollado. En ese sentido, la Unión no solo es la mayor economía del mundo, en algunos casos, es el principal destino de las exportaciones de los países no desarrollados. Para los países donde las exportaciones representan una proporción grande del Producto Interno Bruto (PIB) contar con un número reducido de socios comerciales representa una vulnerabilidad múltiple. La oferta exportable no muy diversificada. Se restringe a un número limitado de bienes básicos, cuyos precios tienden a ser inestables en épocas de crisis. En un mundo más abierto a los flujos de bienes, servicios y capitales, la crisis europea se debe transmitir a la periferia del capitalismo mundial por algunos canales específicos, como ya sucedió entre 2008 y 2009. Además puede interpretarse igualmente como un episodio de propagación a muchas regiones del mundo.

Los problemas asociados con las crisis financieras en general, y con una crisis de deuda en particular, tuvieron consecuencias productivas y sociales catastróficas, con el antecedente histórico vivido en América Latina. En la actualidad, la dirección de las preocupaciones ha cambiado. La pregunta es saber cómo la crisis europea afecta al Tercer Mundo. Durante los años 70, los países no desarrollados vivieron un período de endeudamiento muy acelerado, que originó, en el decenio siguiente, una crisis financiera, económica y social generalizada, conocida como la década perdida. Representó un retroceso sin prece- 
dentes en las posibilidades de desarrollo regional. El afán de aquel entonces era determinar la forma en que se transmitirían sus efectos desde el Tercer hacia el Primer mundo y la eventualidad que se produjera una crisis financiera global. El supuesto desastre que se cernía sobre el sistema financiero internacional se contuvo aplicando medidas de choque, como los programas de ajuste y las reformas estructurales. Algo similar se les exige a los países europeos, especialmente su periferia, en las circunstancias actuales.

Pero aún cuando la Unión sufre los efectos catastróficos de la crisis en términos de crecimiento, empleo y bienestar, las demás economías desarrolladas han corrido la misma suerte si bien con un grado de severidad menor. De acuerdo con la oficina europea de estadísticas, Eurostat, en el año 2012 Estados Unidos apenas creció un 2,2\%, el Reino Unido 0,2\% y Japón 1,9\%, cifras bastante modestas para las principales economías del mundo desarrollado (Eurostat, 2012). En el conjunto de la Unión (27 países) el crecimiento fue negativo $(-0,4 \%)$. Entonces, cuando el mundo desarrollado experimenta problemas económicos, éstos adquieren una connotación global; si a lo anterior se suma la desaceleración China, que es una economía esencialmente exportadora, se acrecientan las probabilidades de efectos adversos sobre las economías del tercer mundo. Son poco diversificadas, vulnerables y dependientes del conocimiento tecnológico y los recursos financieros externos. Para Guillen (2013) la crisis europea es una prolongación de la crisis financiera global de 2007 y a la vez ambas estuvieron imbricadas con la crisis inmobiliaria de los Estados Unidos y de Europa.

\section{Vínculos macro financieros}

A la hora de determinar la forma en que se propaga la crisis de la deuda europea a otras regiones, y de allí a la economía real, la perspectiva de los vínculos macro-financieros enlaza las crisis financieras con las fluctuaciones económicas. De acuerdo con Páez, et. al. (2013), las perturbaciones financieras son la crisis de deuda, la crisis bancaria y las burbujas especulativas. Estas socavan la estabilidad del sector financie- ro y se difunde a otros niveles económicos, a otros mercados o incluso por diferentes países. Conforme a su intensidad, estas crisis se contagian con un aumento significativo de la correlación de la rentabilidad de los activos financieros, cuando se incrementa la volatilidad de sus precios (De Bandt \& Harttman, 2002). La volatilidad se refiere al grado en que los precios de los activos varían a lo largo de un periodo de tiempo, ${ }^{1}$ al grado de fluctuación del precios de los activos, y se mide por la desviación estándar de las series temporales, originada en dichos cambios de precios o en el promedio móvil de una muestra (rolling sample) ocasionada ésta en una mayor varianza del activo.

En consecuencia, la volatilidad es una manifestación de las circunstancias cambiantes del entorno económico, que recoge las oscilaciones del precio de los activos y la reasignación del riesgo entre los agentes económicos. Los períodos de alta volatilidad coinciden con un ciclo económico más pronunciado, de manera que puede hallarse una propagación y un contagio desde las variables financieras a las reales cuando la volatilidad es mayor. El FMI (IMF, por sus siglas en inglés) encuentra una correspondencia entre la volatilidad del precio de los activos y las recesiones económicas (International Monetary Fund, 2003). Esta relación entre los cambios repentinos del precio de los activos con cambios abruptos en la economía real, genera inestabilidades no sólo en el sistema financiero, también tiene implicaciones negativas en la asignación del crédito y la liquidez, con ese potencial grande de producir más turbulencias en la actividad real.

Como los períodos de inestabilidad financiera están asistidos de una mayor volatilidad del mercado, cuando llega a ser extrema (tail events) se convierte en una fuente potencial de tensiones en las instituciones financieras; es decir que la caída de los precios se asocia frecuentemente con fluctuaciones financieras extremas que afectan el rendimiento de los activos. Hay dos tipos de estas perturbaciones. Una de ellas se denomina amplia o sistemática (broad shock) cuando afecta segmentos amplios del siste-

\footnotetext{
Más técnicamente, la volatilidad del precio se define como la desviación estándar de los cambios en el logaritmo de los precios de los activos financieros.
} 
ma financiero. La otra, llamada idiosincrática, se extiende sólo por algunas instituciones específicas; en otras palabras, las oscilaciones de los precios se manifiestan en algunos mercados concretos. Empero, la estructura de correlación de activos financieros no solo es importante para determinar la propagación de las crisis, también lo es para las decisiones sobre la composición de portafolios de inversión y la determinación del riesgo (Kolb, 2011).

Un primer supuesto sobre los vínculos macro-financieros se origina con el modelo Modigliani-Miller (Morley, 2013). Se infiere que no hay una relación entre las variables financieras y las reales, porque las decisiones de inversión se determinan en unas circunstancias macroeconómicas concretas, como el tipo de interés, no por las contingencias en el sector financiero. Sin embargo, debe recalcarse que las empresas se sirven del mercado de capitales y los instrumentos financieros (deuda y acciones) para adelantar los proyectos de inversión, que del mismo modo están sujetos a la volatilidad de los precios en los periodos de turbulencia financiera. La relación dista de ser trivial porque las oscilaciones financieras actúan sobre los recursos disponibles, el patrimonio neto de los agentes, el precio y el tipo de interés de los bonos, de las acciones y la política de dividendos. También de las expectativas de los inversionistas, mientras que el desplome de los índices bursátiles produce grandes pérdidas en el valor de las empresas.

Pero también se han reconocido canales de propagación específicos desde el sector financiero (la mayor correlación de los activos) al real. No obstante, estos canales suponen un movimiento en ambas direcciones. Una crisis real incide sobre las variables financieras que pierden su capacidad de ahorro y de endeudamiento, cortedades que se trasladan al sector financiero, reforzado por la caída en las tasas de ahorro. Al sector real porque cuando se reducen los ingresos de los hogares y los beneficios de las empresas, no pueden reembolsar los créditos al sector financiero, que a la vez experimenta dificultades debido a la contracción de la demanda agregada.

Los canales de transmisión de las crisis financieras al sector real son el equilibro patrimonial de los usuarios del sector bancario (borrower balance sheet channel), el canal asociado con el equilibrio patrimonial de los bancos (bank balance sheet channel) y el canal de liquidez (liquidity channel). A los dos primeros se les refiere como el acelerador financiero. Enfatizan, de acuerdo con (Vousinas, 2013) la influencia que tiene el patrimonio neto (equity position) de los usuarios del crédito y los bancos sobre las condiciones de crédito. Éstas surgen de las fricciones en el mercado de capitales, por la información asimétrica entre bancos y clientes, los mercados incompletos en los que no se satisface una parte de la demanda por crédito, los costos de agencia y el costo que implica forzar el cumplimiento de los contratos.

El canal del crédito enfatiza los efectos de la liquidez sobre los balances bancarios. Subraya las rigideces que se originan cuando se altera esta variable (nominal) porque termina afectando las variables reales. Si las operaciones de crédito son una función del patrimonio neto de usuarios y bancos, la volatilidad compromete el acceso al crédito y la capacidad de irrigar préstamos (Caprio, 2011). Para el Bank of International Settlements BIS (2011), el primer canal por el que transitan las perturbaciones financieras al sector real, se asocia con la posición patrimonial de los usuarios del crédito (borrower balance sheet channel) de su patrimonio neto. Detrás de esta posición subyace la idea de que las instituciones crediticias no pueden evaluar completamente la solvencia y el riesgo de sus clientes. Tampoco pueden hacer un seguimiento exhaustivo a los créditos (monitoreo).

En una crisis, obligar su devolución total. Por estas razones, exigen un respaldo en forma de garantías, representadas en la posición patrimonial, posición decisiva para acceder al crédito. Si la posición patrimonial del usuario es débil o no tiene un patrimonio neto positivo, no es objeto del crédito y se obliga a pagar un costo adicional, la prima financiera externa (external finance premium). Este sobrecosto de la financiación depende inversamente de la solvencia y está estrechamente relacionado con el patrimonio neto. Otra justificación para el cobro de esta prima reside en la predisposición de los usuarios del sector financiero a conseguir créditos cuantiosos que están más allá de sus posibilidades patrimoniales, por lo que asumen un riesgo muy elevado que actúa en detrimento de los intereses bancarios (Bernanke \& Gertler, 1989). 
Estas consideraciones llevan a concluir que las crisis financieras afectan el patrimonio neto de los usuarios del crédito (hogares y empresas). La mayor inestabilidad del sector se traslada a su situación patrimonial a través del acelerador financiero. Transmite mayores correlaciones del precio de los activos y altera el patrimonio neto de los usuarios, sus ingresos $y$ beneficios. Se refleja en una prima financiera externa mayor que incrementa el costo del financiamiento. En una situación de crisis, alguna parte de los activos, el patrimonio neto de hogares y empresas terminan claudicando como garantía de los bancos, que los toman en calidad de pago, situación que se traslada a la demanda agregada. Las perturbaciones financieras se propagan a la producción a través de la prima financiera externa, que amplifica las fluctuaciones de la actividad económica real. Por eso se denomina acelerador financiero.

Holström \& Tirole (1997) examinan igualmente el tema de las garantías. Suponen que hay crédito no intermediado que requiere mayores garantías. Si los usuarios no disponen de ellas, acuden al crédito bancario que es más costoso y limitado, por las restricciones de capital y el monitoreo. En consecuencia, los usuarios sin garantías no acceden al mismo, y en presencia de perturbaciones financieras, la exigencia de garantías eleva el costo del financiamiento y menos usuarios obtienen un crédito.

El segundo canal es el balance contable del banco (bank balance sheet channel). Se divide en dos partes: el crédito y la capitalización. Las crisis financieras deterioran el equilibrio contable de los bancos a través de la política monetaria, el marco regulatorio y la descapitalización. Provocan choques que castigan el balance contable y el crédito, que se difunden a la actividad real, cuando los agentes dependen grandemente del crédito. Si por alguna razón los bancos interrumpen el flujo de recursos, los usuarios buscan alternativas que resultan más costosas, lo que revela que en las crisis financieras hay pérdida de capital para los bancos. De igual forma, un aumento en la correlación de activos por la mayor volatilidad de precios, termina trasladándose a los usuarios que necesitan del crédito. Como las alternativas de financiamiento son escasas y costosas, los usuarios restringen sus gastos en consumo y bienes de in- versión que se trasladan a la demanda agregada, la economía real. Las consecuencias más visibles han sido una contracción global de la demanda y la producción por la restricción del crédito y la caída, sin precedentes, del comercio mundial. Además, como los bancos no pueden aislarse de estas perturbaciones, los choques tienen efectos indeseables sobre su capital.

El canal de liquidez se refiere a la importancia que cobra esta variable en la capacidad de los bancos para intermediar los recursos al sector productivo. Grados de apalancamiento relativamente altos y desajustes en el vencimiento de los pasivos, sacuden los balances bancarios. Son elementos significativos en la propagación de los choques de liquidez para financiar el crédito y la economía real. Durante una crisis financiera, los bancos venden activos que producen un exceso de oferta en el mercado de capitales y los precios se desploman, situación que refuerza aun más la venta de activos pero a precios menores, descapitaliza los bancos, lo que se considera un impacto negativo sobre la liquidez y el nivel de la capitalización. Dados los efectos de interacción y refuerzo, la falta de liquidez ocasiona problemas de solvencia que impide que las instituciones financien activos líquidos con deuda de corto plazo. Se desencadena una crisis bancaria cuando los ahorradores retiran masivamente los depósitos o no están dispuestos a refinanciar las deudas y los bancos se ven obligados a ejecutar embargos y sacrificar otros préstamos más rentables.

El comportamiento de los activos financieros provoca una reacción en cadena, como un comportamiento de manada y desencadena la crisis real; con el cambio de percepción de los agentes económicos, se agudiza la crisis. De acuerdo con Kolb $(2011,5)$ otra causa se asocia con el cambio de percepción sobre la situación de un país aunque no esté afincada en la realidad (wake-up call). Cuando los inversionistas creen que aumenta la probabilidad de ocurrencia de un suceso adverso en el sector financiero en un país específico, aumenta la creencia de que el suceso se disemine a todos aquellos países que comparten características similares. A manera de ejemplo, cuando se creyó que en Grecia se desataría la crisis de la deuda, los inversionistas supusieron que en otros países sucedería 
lo mismo. Efectivamente los episodios con la deuda soberana griega se propagaron a países con características similares, lo que representa un riesgo mayor que termina en pérdidas financieras. Para Kiyotaki \& Moore (1997) los activos financieros desempeñan un doble papel. Con la inversión se producen bienes y servicios pero además se emplean como garantías crediticias. Si el usuario no puede pagar un crédito, sus activos son la garantía de que el banco no pierde totalmente los recursos. Entonces cualquier perturbación que reduzca el valor de los activos e introduzca volatilidad de los precios, afecta el patrimonio neto de los agentes y limita el monto de los créditos. Supone de igual manera, una prima de financiamiento externo mayor. La restricción del crédito afecta la producción de bienes y servicios y rebaja aun más el precio, el valor de los activos financieros, los ingresos de los hogares y los beneficios de las empresas, trastornando considerablemente la actividad real.

Cuando una crisis financiera deteriora la posición patrimonial de los agentes económicos y las condiciones del crédito, da lugar a problemas recurrentes en el sector real, pero también los agentes enfrentan otras dificultades no menos complejos, como las asimetrías de información, el incumplimiento de contratos y los costos de agencia. Bajo el supuesto que el sector financiero conoce la naturaleza y las particularidades del mercado, sus decisiones, apoyadas en ese conocimiento, tienen efectos, algunos de ellos indeseables, como la manipulación de los precios de los activos, las burbujas especulativas y la creación de derivados por fuera de cualquier regulación.

La expectativa de beneficios extraordinarios apostándole al aumento del precio y demanda de un activo actúa sobre el comportamiento de los inversionistas que asumen un riesgo exagerado, estrategias especulativas que conducen a la bancarrota financiera y, eventualmente, compromete la estabilidad del sistema financiero internacional. Otro vínculo muy dinámico se encuentra entre el mercado de capitales y la política monetaria (Laopodis, 2009). El desorden en el mercado de capitales puede inducir la intervención del banco central, con resultados adversos para el crecimiento económico, sobre las decisiones de ahorro e inversión de los agentes por la vía de las fluctuaciones del tipo de interés.

\section{Vulnerabilidad de los países del Tercer Mundo a la crisis europea}

La crisis financiera global, después de un periodo de recuperación bastante frágil para algunos países a partir de 2010, entró en una nueva fase recesiva, materializada en la crisis europea actual. Ésta última es una prolongación de la primera. Con posterioridad al repunte observado en 2010, cuando el comercio global se expandió a una tasa cercana al 14\% en volumen, en 2011 se redujo al 5\% (WTO, 2013). Para 2013, esta organización prevé una expansión de solo un $3,3 \%$ en volumen, inferior al promedio anual de $5.3 \%$ observado durante las últimas dos décadas. De acuerdo con la CEPAL, se debe principalmente a las repercusiones de la crisis europea (CEPAL, 2012).

La actividad comercial continúa siendo lenta en los primeros meses del año 2013, atribuible a la contracción de la demanda en Europa y el crecimiento errático en las economías no desarrolladas. Para la WTO (2013), el crecimiento del comercio internacional durante los últimos 20 años fue cercano al 7\% anual en términos brutos (valor en dólares o dollar value) alcanzando la extraordinaria cifra de 18 billones de dólares al final del período, hecho imputable a la reducción de aranceles y otras barreras al comercio internacional.

Por las repercusiones globales que alcanzan la crisis financiera y la crisis de la deuda europea, es apenas admisible que los países del Tercer Mundo no pueden pasar indemnes. En ese sentido, se han identificado algunos canales de transmisión específicos de las perturbaciones financieras observadas en Europa sobre la actividad económica y por consiguiente al sector real. Entre estos se encuentran el canal comercial, el canal financiero, el acceso al crédito para financiar necesidades presupuestales (deuda), las remesas de los inmigrantes y la ayuda para el desarrollo o cooperación internacional.

Con respecto al canal comercial, éste se refiere al comercio internacional y más específicamente a las exportaciones desde el Tercer Mundo. El punto reviste una importancia analítica notable, porque la Unión Europea es, junto con Estados Unidos y China, el principal destino de las exportaciones desde el Ter- 
cer Mundo empero, no están muy diversificadas. En 2012, el principal destino de las exportaciones de América Latina y el Caribe fueron Estados Unidos con el $41 \%$, Asia $17 \%$ y la Unión Europea el 13\%, para un total de $71 \%$ (CEPAL, 2012, 5). Para África, más del $60 \%$ de las exportaciones tuvieron como destino la Unión Europea y Estados Unidos. Con respecto a la concentración de los bienes exportados, el $57 \%$ de países africanos sólo 5 productos dan cuenta del $75 \%$ de las exportaciones y en los casos más críticos, en el $15 \%$ de ellos un sólo producto representa el $75 \%$ de sus exportaciones. El índice de concentración de Herfindahl es 2811, es decir, el destino de las exportaciones está concentrado en un número muy reducido de mercados. El otro punto

Ilustración 1. Crecimiento Económico Mundial, PIB Real, 2000-2011 3

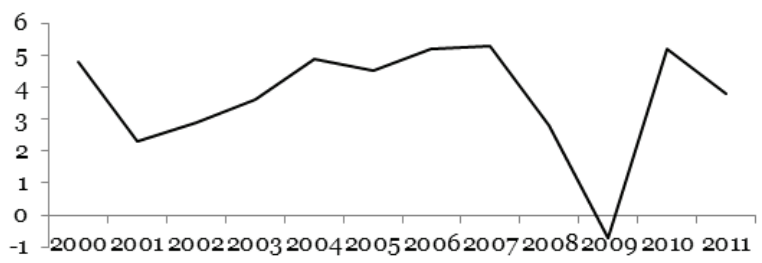

Ilustración 3. Exportaciones del Tercer Mundo ${ }^{5}$

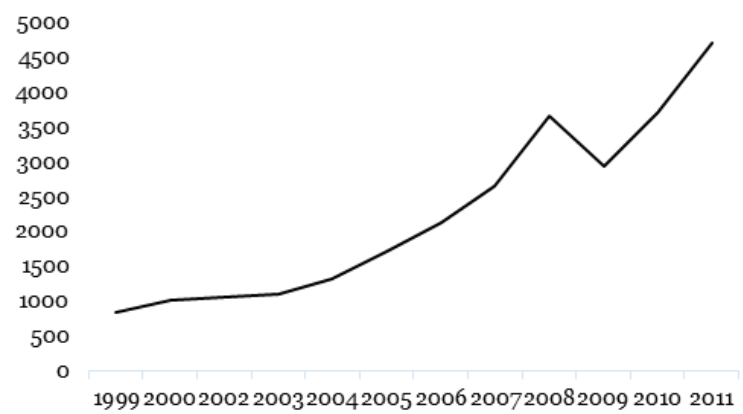

de interés es que una gran proporción de las exportaciones son bienes primarios, cercano al 70\% (FMI, 2009). Los destinos y las exportaciones están muy poco diversificados.

La dependencia de pocos socios comerciales y la concentración de las exportaciones en bienes mayoritariamente primarios ${ }^{2}$, acentúa las vulnerabilidades en los periodos de crisis, con la eventualidad que aumente la volatilidad de los precios de las exportaciones, esencialmente a la baja. En estas circunstancias hay una menor demanda de bienes del Tercer Mundo que resulta ser pro-cíclica, con implicaciones negativas para los precios y los volúmenes, una elasticidad renta muy elevada y un marcado deterioro

Ilustración 2. Crecimiento Económico Mundial, PIB Real, 2000-20114

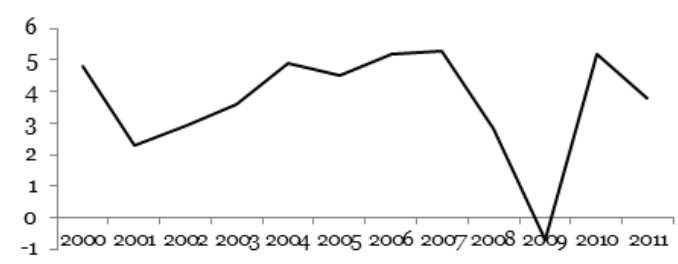

Ilustración 4. Exportaciones promedio Tercer Mundo $1999-2011^{6}$

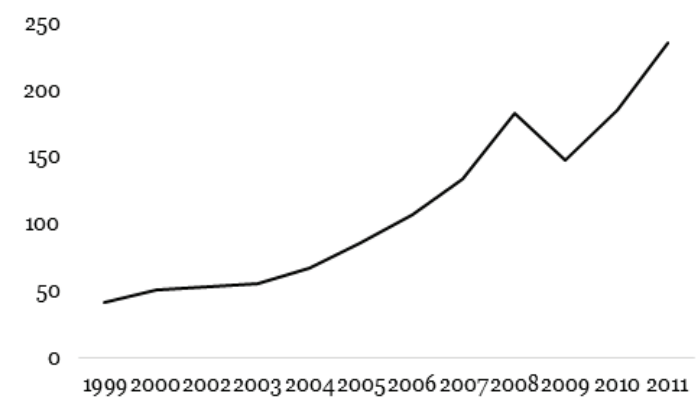

2 También se denominan bienes básicos, materias primas o commodities. Aquí se usan estos nombres indistintamente.

3 Fuente: Elaboración propia con base en OECD (2012) e Index-mundi. Las exportaciones se definen como el monto total en dólares de Estados Unidos de las exportaciones de mercancías FOB y se calculan al tipo de cambio corriente. Datos para Chile, Camboya, China, Colombia, Argentina, Brasil, Grecia, India, Indonesia, México, Namibia, Pakistán, Perú, República Democrática del Congo, Rusia, Singapur, Sri Lanka, Sudáfrica, Tailandia.

4 Fuente: Elaboración propia con base en OECD (2012) e Index-mundi.

5 Fuente: Elaboración propia con base en OECD (2012) e Index-mundi.

6 Fuente: Elaboración propia con base en OECD (2012) e Index-mundi. 
de los términos de intercambio. Mientras el precio de las exportaciones de un país se deteriora, el precio de las importaciones se mantienen constantes. $\mathrm{El}$ primero debería aumentar el volumen de las exportaciones para compensar la caída de los precios de exportación.

Con la crisis europea, las prioridades en materia económica están orientadas a mejorar la situación en su área de influencia. Esto perturba otro canal financiero muy importante como la inversión extranjera directa (IED) y la muy cuestionada ayuda para el desarrollo. De manera que en su conjunto, los componentes del canal financiero transmitidos a partir de una crisis financiera, tienen efectos indeseables para el crecimiento económico de los países no desarrollados, bien porque no tienen los recursos financieros y técnicos para explotar los recursos o porque esta falta impide la acumulación de capital físico (formación bruta de capital). La falta de recursos impide o retrasa algún tipo de crecimiento, si así puede llamarse a la esquizofrenia de la teoría económica de que la razón de ser de una economía es el crecimiento per sé.

Para una muestra de 20 países del tercer mundo ${ }^{7}$ en el periodo comprendido entre 1999 y 2011 se contrastaron los datos de la tasa de crecimiento económico global para el período 2000-2011 y la tasa de crecimiento del valor de las exportaciones (dollar value). Las ilustraciones 1 y 2 dejan ver palmariamente la pro-ciclicidad de las exportaciones del Tercer Mundo. Cuando se contrae la producción mundial, las exportaciones siguen un patrón marcadamente equiparable, pues se reduce la demanda de bienes básicos con este origen geográfico. Otro problema asociado con los anteriores, es la caída de los precios de las materias primas y en consecuencia el deterioro de los términos de intercambio, con unos efectos fiscales considerables.

En presencia de una crisis financiera en los países que son destino de las exportaciones, disminuye la demanda porque sus habitantes y los mismos gobiernos compran menos. A menudo una contracción de la renta en los países importadores tiene un efecto magnificado sobre la demanda más que proporcionado, una elasticidad renta de las exportaciones mayores a la unidad.

Como en los países en crisis se incrementa el desempleo (es anti-cíclico) los segmentos de población desocupada se queda sin recursos (no tienen ingresos). La demanda, se ve aun más disminuida por los recortes de los presupuestos públicos que se manifiestan en las medidas de austeridad. Si la crisis alcanza proporciones inusuales, con el rescate del sistema financiero $e$ incluso de países enteros, se vulnera el trabajo de los inmigrantes. Con la crisis pierden los empleos y con ellos una merma de las remesas que envían a sus países de origen.

La ilustracion 1 muestra la magnitud de las dificultades que experimentan las exportaciones ante un colapso de la economía mundial. La tasa de crecimiento de la producción fue negativas en 2009, pero a partir de allí se inicia una recuperación modesta. Luego sobreviene con todo su rigor la crisis europea, consecuencias que se empiezan a sentir a partir del 2011, a lo largo del 2012 y 2013. Las exportaciones desde el Tercer Mundo siguen una tendencia análoga que muestra la pro-ciclicidad del comercio internacional. En la ilustración 2 y al final del periodo las exportaciones no se han descolgado completamente, pero esto sucede en 2012 como se discutió en la parte precedente. Las ilustraciones 3 y 4 muestran las tendencias de las exportaciones de largo plazo por su valor en dólares y el promedio, donde se corroboran estas consideraciones. Las tendencias dejan entrever un crecimiento sostenido de largo plazo en el valor de las exportaciones pero también el efecto desfavorable de las crisis sobre las exportaciones del TM.

Un segundo canal de transmisión de la crisis europea al Tercer Mundo es el canal financiero. Para los países de ingresos bajos, los mercados de capitales son reducidos, por lo que de alguna manera se aíslan de choques financieros con las proporciones observadas en el Primer Mundo. En consecuencia, este canal adquiere unas connotaciones particulares, $y$

Chile, Camboya, China, Colombia, Argentina, Brasil, Grecia, India, Indonesia, México, Namibia, Pakistán, Perú, República Democrática del Congo, Rusia, Singapur, Sri Lanka, Sudáfrica, Tailandia. 
se asocia con una disminución considerable de los flujos de capital originados en los países europeos. Los componentes del canal financiero son la inversión extranjera directa (IED), las remesas, la ayuda económica para el desarrollo o cooperación internacional y el acceso al financiamiento en los mercados internacionales de crédito.

Si se analiza la relación entre crisis y el canal financiero, es claro que la inversión es uno de los factores más golpeados pero más necesarios para el crecimiento de los países más pobres y atrasados. Pero al igual que con las exportaciones, estos componentes se ven disminuidos, de acuerdo con las tendencias observadas con la IED, la ayuda para el desarrollo y las remesas. No obstante que son una fuente significativa de recursos para financiar inversiones productivas y la generación de empleo en los países receptores, durante una crisis en el centro, esta fuente de recursos presentan una gran inestabilidad.

La ilustración 5 muestra la evolución de la IED hacia los países no desarrollados en diferentes años. Durante la década pérdida y en una situación que se prologa hasta el año 2000, los flujos de inversión extranjera directa se dan principalmente entre economías del norte, valga decir, economías desarrolladas. Los flujos adquieren la dirección norte-norte. La situación cam-

Ilustración 5. Distribución de la IED años seleccionados ${ }^{8}$

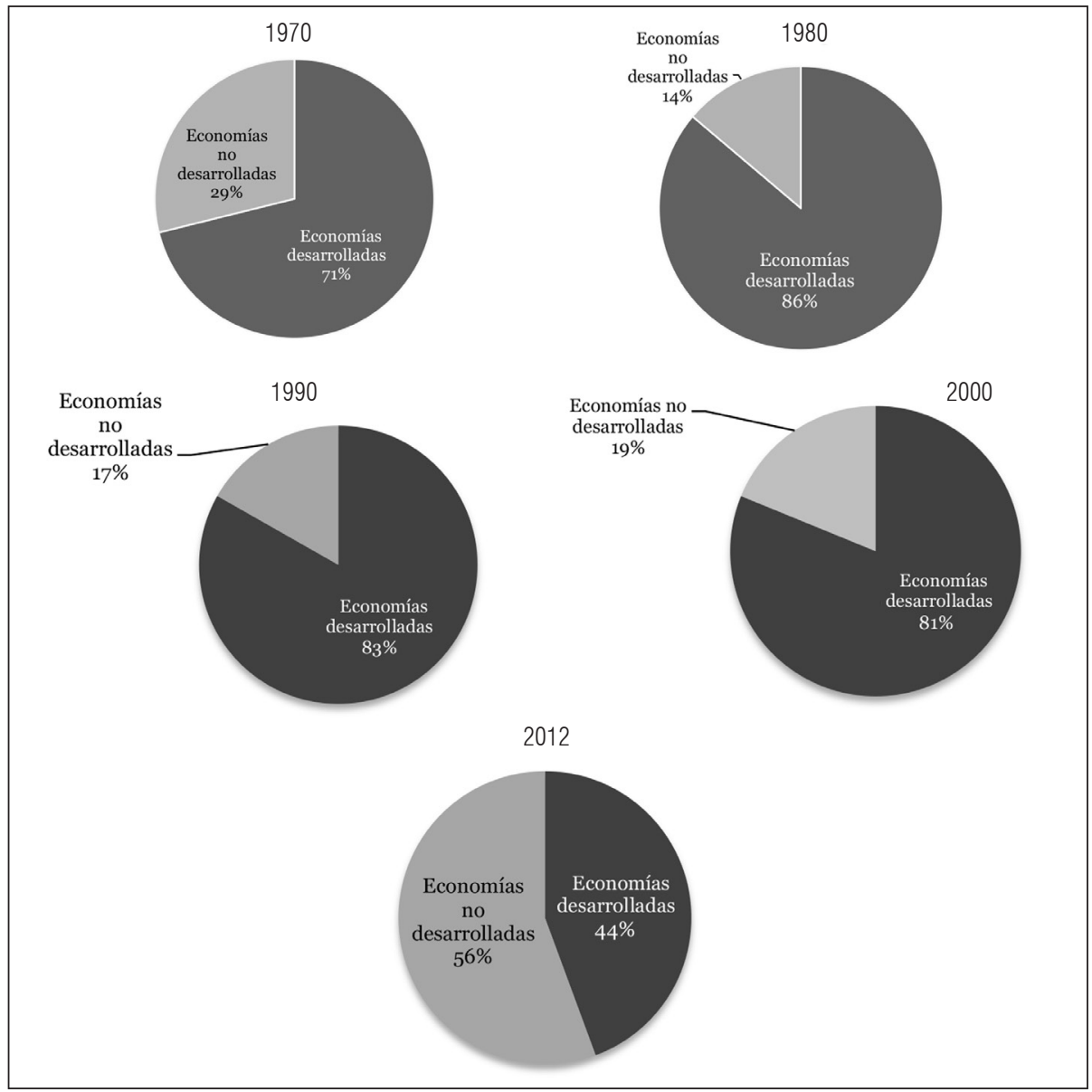

8 Fuente: Elaboración propia con base en UNCTAD (2013). 
bió drásticamente en 2012. Esto es el resultado de una mayor movilidad del capital, toda vez que, a la luz de las reformas institucionales que acompañaron los programas de ajuste y las reformas estructurales, se moderó la regulación y se crearon condiciones onerosas para los países receptores de la IED. Estos cambios favorecieron a las empresas, especialmente transnacionales. La menor regulación y la toma hostil de empresas públicas y privadas con las privatizaciones, las fusiones y adquisiciones, las garantías de rentabilidad mínima, la onerosa exigencia de estabilidad jurídica y la exención de impuestos, amén de otras concesiones que hacen los gobiernos del Tercer Mundo, se verá reflejado en un crecimiento sin precedentes de la IED, alimentada por la expansión del comercio y la producción y la extracción de recursos naturales, abundantes en amplias regiones del mundo no desarrollado, explotación que se ve favorecida por la expansión dinámica de la demanda en el centro. Otro elemento no menos importante en la expansión de la IED está representado por la decisión de los inversionistas de trasladar la producción desde los países desarrollados, donde la mano de obra y otros factores productivos son costosos, hacia países no desarrollados donde son abundantes y baratos.

Pero aun así, desde una perspectiva de más largo plazo, si bien el valor en dólares de la IED creció en promedio desde 1970, también muestra unas oscilaciones muy marcadas que coinciden con los periodos de expansión y crisis de la producción global. La ilustración 6 muestra cómo el valor de la inversión extranjera fue afectado durante la crisis financiera global, aunque los datos sugieren que si bien los flujos se contraen en el agregado, la disminución es más dramática entre economías desarrolladas. Las economías no desarrolladas también ven disminuir el valor de la inversión, si bien menos que proporcionalmente. Es indudable la contracción a partir de la crisis europea.

De acuerdo con los datos de la UNCTAD (2013), y representadas en las figuras 6 , se corrobora una coincidencia entre desempeño económico y el signo de la tasa de crecimiento de la IED.
Ilustración 6. Valor en dólares de la IED 1970-20129

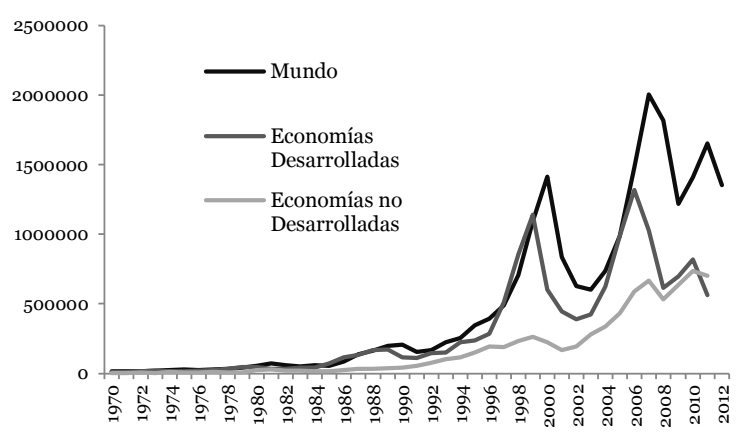

Durante la primera parte de los 70 los flujos de recursos hacia el TM presentan un signo negativo. La contracción coincide con la primera crisis del petróleo; aun cuando los flujos de IED no fueran muy significativos para el TM, no alcanzaron a tener signo negativo. La IED empieza a ser importante durante los 80's en el contexto más amplio de la globalización, aunque es indiscutible que durante una crisis resulta ser pro-cíclica. Crecimientos de la economía mundial ocasiona el aumento de la IED en los países desarrollados y no desarrollados, pero lo contrario resulta ser significativo. La IED es negativa durante la crisis de la deuda y definitivamente se desploman en la crisis del 1982 y 2003, la crisis financiera global y las crisis europeas actuales, que muestra una disminución significativa.

Ilustración 7. TCR PIB 1970-2012

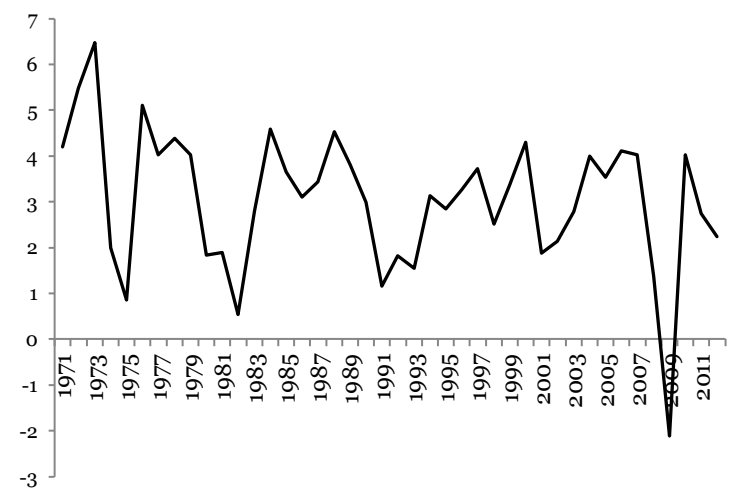

Fuente: Cálculos propios con base en UNCTAD. UNCTAD Statistics (2013).

10 Fuente: Elaboración propia con base en UNCTAD (2013) 
Ilustración 8. Tasa de crecimiento IED 1970-2012 ${ }^{11}$

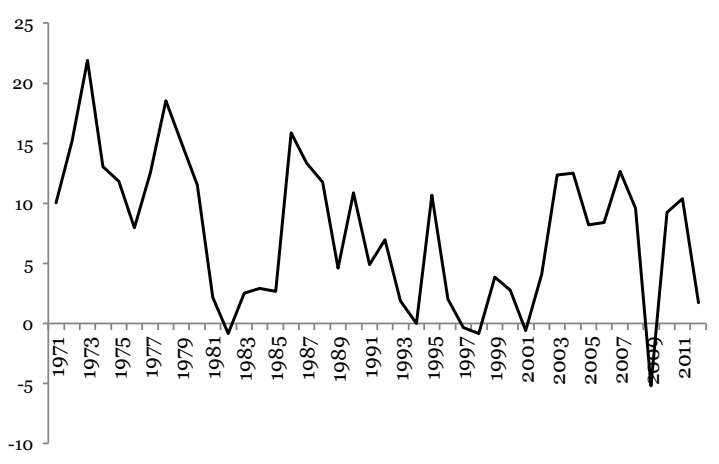

Según Poulsen \& Hufbauer (2011) en 2007 la IED alcanzó un valor máximo histórico de unos 2 billones de dólares ${ }^{12}$; hasta allí predomina la tendencia creciente de estos flujos. Es decir que con la crisis financiera global, los flujos globales de IED comenzaron a contraerse, un $16 \%$ en 2008 , y cuando la producción mundial se contrajo en 2009 por primera vez en 60 años, la inversión extranjera directa se redujo un 40\%. Durante el año 2010 los niveles de inversión extranjera directa se estancaron por encima de un billón de dólares.

La caída de la IED se puede atribuir a otros factores asociados con la crisis y la situación de las empresas (UNCTAD, 2009) pero originadas también en la crisis financiera global, y en esa misma dirección, la crisis europea. A través del canal financiero se transmiten las restricciones de liquidez para las empresas transnacionales (ETN); se restringe el acceso al crédito y se endurecen las condiciones para acceder, por el deterioro de la tasa de beneficio de las empresas y porque la capacidad de éstas para invertir se debilita considerablemente. En segundo lugar, el vínculo entre crecimiento y flujos de IED significa que la crisis mundial que afecta al mundo desarrollado, ha reducido el deseo de las empresas europeas para sostener el ritmo de inversiones en el extranjero. Probablemente ha fomentado una actitud más cautelosa entre éstas, lo que resulta en el abandono de proyectos de alto riesgo hacia activos más seguros, como bonos públicos con un riesgo mínimo y menor rentabilidad.

11 Fuente: Elaboración propia con base en UNCTAD (2013)

12 Millones de millones.
Con una expansión de la producción mundial, la IED se mueve en la misma dirección y al contrario, una crisis global implica la contracción severa de la inversión. La misma tendencia se observa en las economías no desarrolladas. Se puede concluir que las crisis financieras trasladadas al sector real, se traducen en menores tasas de crecimiento y un aumento del desempleo, junto con las medidas de austeridad que contrae los presupuestos públicos en servicios ciudadanos con un efecto negativo sobre la IED para todos los grupos de países.

Otro componente muy importante del canal financiero son las remesas, que después de la inversión extranjera directa, son la segunda fuente de financiamiento externo para ciertos países del Tercer Mundo, (Albo \& Díaz, 2011). De acuerdo con La ilustración 7, los principales países receptores de las remesas son India, China, Filipinas y México, todos ellos del TM. Una contrastación de las ilustraciones 7 y 8 deja entrever una relación interesante entre el monto de la remesas, el tamaño de la economía y la importancia relativa de éstas como proporción del PIB. Los países mencionados son los principales receptores de remesas y al mismo tiempo son economías comparativamente grandes, especialmente China, India y México, de manera que las remesas vienen a ser una proporción pequeña del PIB.

En la medida en que las remesas son una proporción muy grande del PIB de un país frente a otros componentes, las economías tienden a ser más pequeñas y menos diversificadas. Probablemente dependen mucho más de las materias primas y pocos socios comerciales también. A pesar que las economías reseñadas reciban una gran cantidad de dinero por concepto de remesas. Por ejemplo, a India ingresaron US\$ 70 mil millones durante 2012 pero son apenas el 3,8\% del PIB, mientras que para China es el 0,7\% con US\$ 66 mil millones (World Bank, 2012).

Para otros países, como se muestra en La ilustración 10 , son una proporción mayor del PIB. Entonces no se puede hacer un análisis de las remesas y su efecto sobre un país sin tener en cuenta esas par- 
ticularidades. Entre más modesta y poco diversificada sea una economía, es más probable que las remesas sean decisivas a la hora de determinar su desempeño social y macroeconómico; y son más vulnerables a los vaivenes de las remesas. Tienen así también un componente pro-cíclico, aumentando así los ingresos durante los auges y depresión durante las recesiones.

Ilustración 9. Remesas en miles de millones de dólares, $2012^{9}$

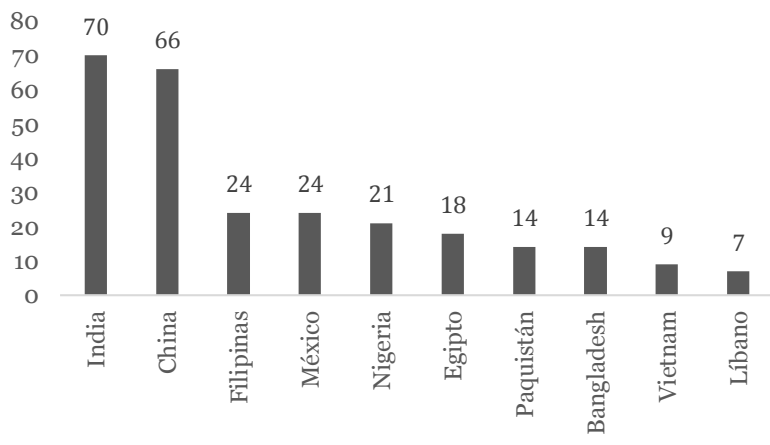

llustración 10. Remesas como \% PIB ${ }^{10}$

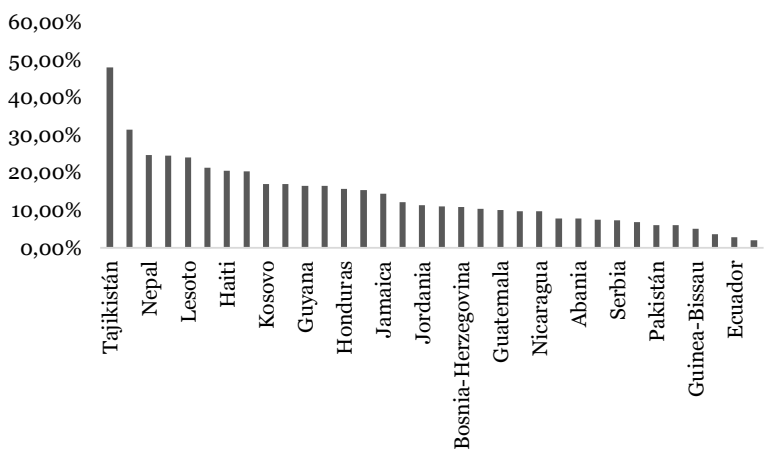

La ilustración 11 muestra el patrón de las remesas hacia las economías no desarrolladas (tasa de crecimiento en dólares corrientes, tipo de cambio corriente) a un nivel agregado. Al igual que con las demás variables consideradas, éstas tienden a estar fuerte-
Ilustración 11. Tasa de Crecimiento Remesas. Economías no Desarrolladas ${ }^{11}$

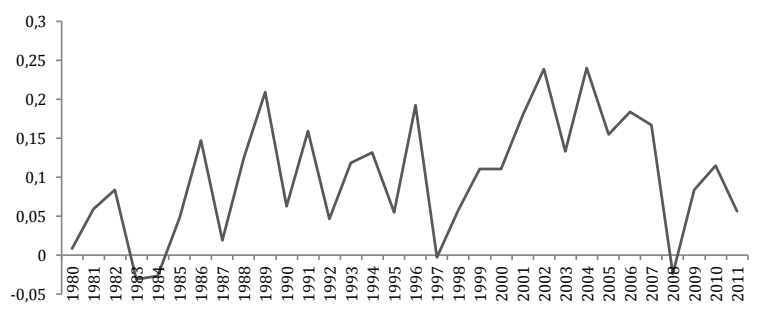

mente correlacionadas con las tasas de crecimiento del PIB. Muestran periodos de contracción bastante agudos como en el periodo 1983-1984 en plena crisis de la deuda externa, lo que ocurre nuevamente en 1987, 1997, 2008 y están reduciéndose considerablemente ahora con la crisis europea. Aunque han crecido en el tiempo, y sus principales destinatarios son los países no desarrollados, la dilación de la crisis en el viejo continente ha desacelerado el flujo de recursos especialmente hacia algunas regiones muy específicas de Europa, Asia Central, África Subsahariana, América Latina y el Caribe. De acuerdo con los datos, puede entreverse que para los países más pobres las remesas constituyen una proporción importante del PIB, por lo que las oscilaciones de las mismas tienen efectos muy grandes sobre la economía que recibe las remesas. El impacto económico y social puede ser considerable y por lo mismo deben ser muy vulnerables a una crisis como la experimentada en Europa y Estados Unidos. Eso lleva a mirar el hecho de que economías grandes y diversificadas inclusive más complejas, están menos expuestas a los vaivenes de las remesas. Cuanto más importantes son las remesas como porcentaje del PIB una crisis que las disminuya tiene efectos macroeconómicos grandes, mientras que para economías más grandes y diversificadas son menos dependientes de las remesas. Es posible considerar que no se vean afectadas como totalidad, pero si aquellas regiones específicas donde la salida de residentes es mayor.

11 Fuente: Elaboración propia con base en UNCTAD (2013). 
Las remesas hacia México se originan principalmente en Estados Unidos, de manera que han sido más propensas al comportamiento de esta economía y la contracción está más afectada por la crisis de las hipotecas o la burbuja inmobiliaria de 2007. En consecuencia, las remesas han disminuido su importancia económica en algunos estados como Oaxaca, Guerrero e Hidalgo pero su importancia sigue siendo alta en los ingresos de Michoacán y Zacatecas (Albo, et. al. 2011).

Ilustración 12. Tasa de crecimiento de la Ayuda Económica $1960-2011^{1}$

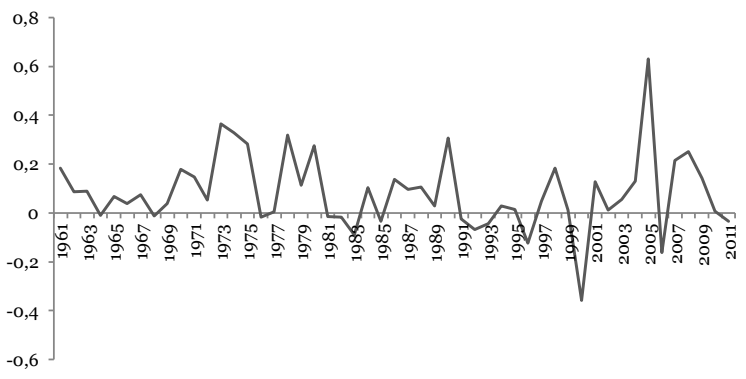

La ayuda oficial para el desarrollo (ODA, por sus siglas en inglés) es otro componente del canal del crédito y una de las fuentes más importantes de financiación externa para el desarrollo, que igual ha disminuido. Proporcionalmente es mucho menor que los montos alcanzados por las exportaciones $y$ la IED (Tabla 1 y 2). Además la Tabla 2 muestra que la distribución porcentual de la ODA por regiones disminuyó a consecuencia de las medidas de aus-
Tabla 2. Principales receptores de Ayuda (Bruta) $)^{14}$

\begin{tabular}{|c|c|}
\hline Principales receptores de Ayuda (Bruta) & US\$ Millones \\
\hline Turquía & 541 \\
\hline Margen Occidental y Franja de Gaza & 490 \\
\hline Afganistán & 340 \\
\hline Congo, RD & 299 \\
\hline Kosovo & 298 \\
\hline Serbia & 292 \\
\hline Sudán & 255 \\
\hline Marruecos & 253 \\
\hline Etiopía & 220 \\
\hline Mozambique & 199 \\
\hline
\end{tabular}

Tabla 3. ODA Neta ${ }^{15}$

\begin{tabular}{|c|c|c|c|}
\hline ODA Neta & $\mathbf{2 0 0 9}$ & $\mathbf{2 0 1 0}$ & Cambio \% \\
\hline Corriente (US\$ Millones) & 13444 & 12679 & $-5,7$ \\
Constantes (Dólares de 2009) & 13444 & 13226 & $-1,6$ \\
En millones de Euros & 9654 & 9573 & $-0,8$ \\
\hline
\end{tabular}

teridad y las presiones presupuestales de los países de Europa.

Desde 2010, año en que alcanzó su punto máximo, la ODA disminuyó un -6,0\% en términos reales. Excluyendo 2007, que supuso el fin de las operaciones de alivio a la deuda, la caída de 2012 es la mayor desde 1997. Esta es también la primera vez desde 1996 hasta 1997 la ayuda se ha reducido en dos años sucesivos.

Tabla 1. Ayuda Europea para el Desarrollo, $2010^{13}$

\begin{tabular}{|c|c|c|c|c|c|c|}
\hline \multicolumn{7}{|c|}{ Ayuda Europea para el Desarrollo, 2010 } \\
\hline \multicolumn{7}{|c|}{ Millones de Dólares. Participación Porcentual } \\
\hline $\begin{array}{c}\text { África } \\
\text { Subsahariana }\end{array}$ & Europa & $\begin{array}{c}\text { Oriente Medio y África } \\
\text { del Norte }\end{array}$ & Sin Especificar & $\begin{array}{c}\text { Asia Central y } \\
\text { del Sur }\end{array}$ & $\begin{array}{c}\text { Latinoamérica y el } \\
\text { Caribe }\end{array}$ & $\begin{array}{c}\text { Otros Asia y } \\
\text { Oceanía }\end{array}$ \\
\hline $37 \%$ & $17 \%$ & $12 \%$ & $11 \%$ & $10 \%$ & $9 \%$ & $4 \%$ \\
\hline
\end{tabular}

\footnotetext{
Fuente: Elaboración propia con base en UNCTAD (2013)

Fuente: Elaboración propia con base en OCDE (2012).

Fuente: OCDE (2012).

Fuente: OCDE (2012).
} 


\section{Conclusiones}

La crisis europea puede entenderse como una extensión de la crisis financiera global. La crisis en el Viejo Continente se inicia en Grecia y se propaga luego a otros países de la periferia europea. El resultado más visible, desde el punto de vista económico, es un desplome de la producción, medida por la tasa de crecimiento del PIB real y el desempleo. En algunos casos la tasa de desempleo superó los dos dígitos. En los casos más extremos se acercan al 30\% de la oferta laboral. La crisis europea tiene efectos sobre las economías del Tercer Mundo, que se transmiten por lo menos en dos niveles. De lo financiero a los financiero y de los financiero a lo real. En el primer caso, las crisis financieras (bancarias, de deuda, burbujas especulativas) se contagian y propagan a otros mercados financieros con el aumento de la correlación de la rentabilidad de los activos financieros, originada está en la mayor volatilidad de los precios.

Luego afecta los sectores financieros de otros países. La crisis, y por ende, la volatilidad en la rentabilidad y la mayor correlación de activos, afecta la posición patrimonial neta de los agentes económicos, obligándolos a asumir un sobrecosto en los créditos, la prima financiera externa. Esto encarece el acceso al crédito cuando la crisis afecta el patrimonio neto, los canales del crédito, la demanda de consumo de los hogares, y de inversión de las empresas, con un efecto negativo sobre el sector real, involucrando otros sectores económicos en diferentes países y regiones.

En relación con el Tercer Mundo, se han identificado canales específicos de transmisión de la crisis. Estos canales son el comercial y el financiero. El primero de ellos se refiere esencialmente a las exportaciones desde el tercer mundo, que adquieren unas características particulares. El destino de las mismas es muy restringido. Cuentan con muy pocos socios comerciales. Por otro lado, están muy poco diversificadas. La primera gran conclusión de la investigación es que ante la crisis europea se reducen las exportaciones del tercer mundo. Esto puede ser bastante problemático para aquellos países que dependen de pocos bienes exportables, generalmente bienes básicos, pocos socios comerciales y las exportaciones constituyen una proporción grande del PIB. Este grupo de países es muy vulnerable a una crisis como la europea.

La crisis europea ha significado también la contracción de la inversión extranjera directa a países del tercer mundo. Y por último, las remesas se pueden ver seriamente comprometidas por las mismas razones y como se vio, también son una fuente de recursos muy importante para las economías no desarrolladas y porque ante el aumento del desempleo en Europa, contrae las posibilidades de migración, de manera que aumentan las tasas de desempleo, el crecimiento económico, con aumentos importantes de las tasas de pobreza absoluta e indigencia.

La crisis de deuda soberana europea tiene repercusiones para el TM, especialmente para los países de renta baja, y sobre sus posibilidades de desarrollo. Puede inducir una crisis de desarrollo. Los recursos financieros dedicados a apoyar a los países no desarrollados disminuyen en el evento de una crisis financiera y económica global como la experimentada hasta estos momentos en Europa y sobre todo si la ocurrencia de estas crisis se hace más recurrente.

\section{Referencias}

Albo, A. \& Díaz, J. L. (2011). Migración Mexicana hacia los Estados Unidos: Una breve Radiografía. Documentos de trabajo. México: BBVA Research.

Bernanke, B. \& Gertler, M. (1989). Agency Costs, Net Worth, and Business Fluctuations. En: American Economic Review, 79(1): 14-31.

Bank for International Settlements - BIS (2011). The Transmission Channels Between the Financial and Real Sectors: A Critical Survey of the Literature. Basel: Bank for International Settlements.

Caprio, G. (2011). Macro-Financial Linkages in IMF Research (I. E. Office, Trans.). Washington: IEO, International Monetary Fund.

Comisión Económica para América Latina y el Caribe CEPAL (2012). Boletín Estadístico: Comercio Exterior de Bienes en América Latina y el Caribe. Santiago de Chile: Comisión Económica para América Latina y el Caribe.

De Bandt, O. \& Harttman, P. (2002). Systemic Risk: A Survey Working Paper Series: European Central Bank.

Guillén, A. (2013). La Crisis Europea: Una Prolongación de la Crisis Global. En: Revista del CIECAS-IPN, VIII(29): 23-41.

Holström, B. \& Tirole, J. (1997). Financial Intermediation, Loanable Funds, and the Real Sector. En: The Quarterly Journal of Economics, 112(3): 663-691.

Fondo Monetario Internacional - FMI. (2009). World Economic Outlook. Washington, D.C: Fondo Monetario Internacional (FMI).

Kiyotaki, N. \& Moore, J. (1997). En: Credit Cycles Journal of Political Economy, 105(2). 
Kolb, R. W. (Ed.). (2011). Financial Contagion: The Viral Threat to the Wealth of Nations. Hoboken: John Wiley and Sons.

Laopodis, N. (2010). "Dynamic Linkages Between Monetary Policy and the Stock Market". En: Review of Quantitative Finance and Accounting, 35(3): 271-293.

Morley, J. (2013). Macro-Finance. Linkages.

OECD (2012). Total DAC Flows at a Glance. Paris: Organization for Economic Development an Cooperation. http://www.oecd. org/dac/stats/totaldacflowsataglance.htm.

Páez, P., Isaza, J. \& Zamora, A. (2013). La Crisis Europea Actual. En: Revista de Relaciones Internacionales: Estrategia y Seguridad, 8(2): 15-36.

Poulsen, L. \& Hufbauer, G. (2011). Foreign Direct Investment in Times of Crisis Working Paper Series. Washington: Petersen Institute for International Economics.
United Nations Conference on Trade and Development - UNCTAD. (2009). Assessing the Impact of the Current Financial and Economic Crisis on Global FDI Flows: United Nations Conference on Trade and Development.

UNCTAD (2013). UNCTAD Statistics. Geneve, United Nations Conference on Trade and Development.

Vousinas, G. L. (2013). The Transmission Channels between Financial Sector and Real Economy in Light of the Current Financial Crisis: A Critical Survey of the Literature. En: Modern Economy, (4): 248-256.

World Bank (2012). Migration and Development Brief No. 19. Migration and Remittances Unit, Development Prospects Group, Washington.

World Trade Report - WTO (2013). World Trade Report 2013: Factors Shaping the Future in World Trade. Geneva: World Trade Organization 